\title{
ECOLOGY AND SOCIETY
}

Home | Archives | About | Login | Submissions | Notify | Contact | Search

\section{ES Home > Vol. 1, No. $1>$ Art. 7}

Copyright (C) 1997 by The Resilience Alliance ${ }^{*}$

Ludwig, D., B. Walker, and C. S. Holling. 1997. Sustainability, stability, and resilience. Conservation Ecology [online]1(1): 7. Available from the Internet. URL: http://www.consecol.org/vol1/iss1/art7/

A version of this article in which text, figures, tables, and appendices are separate files may be found by following this link.

\section{Insight}

\section{Sustainability, Stability, and Resilience}

Donald Ludwig ${ }^{1}$, Brian Walker ${ }^{2}$, and Crawford S. Holling ${ }^{3}$

\section{${ }^{1}$ Department of Mathematics, University of British Columbia; ${ }^{2}$ Division of Wildlife and Ecology, CSI RO; ${ }^{3}$ Department of Zoology, University of Florida}

- Abstract

- Introduction

- A Simple Prototype

- Hard Loss of Stability and Hysteresis

- A Competitive Grazing System

- Fire in a Savanna System

- Concluding Remarks

- Responses

- Literature Cited

The purpose of this essay is to define and refine the concepts of stability and resilience and to demonstrate their value in understanding the behavior of exploited systems. Some ecological systems display several possible stable states. They may also show a hysteresis effect in which, even after a long time, the state of the system may be partly determined by its history. The concept of resilience depends upon our objectives, the types of disturbances that we anticipate, control measures that are available, and the time scale of interest.

KEY WORDS: bifurcation; multiple stable states; resilience; stability. 


\section{I NTRODUCTI ON}

Humans are dependent upon natural systems for the necessities of life such as air and water, as well as resources that are essential for modern societies (Odum 1993). As humans have imposed greater and greater demands upon natural systems, Arrow et al. (1995) and many others have raised concerns about the sustainability of the resource flows from these systems. The purpose of this exposition is to review some theoretical concepts and present specific examples to illustrate the variety of possible behaviors that natural systems may display under exploitation. The concepts stem from our informal understanding of the ideas of stability, sustainability, and resilience, but clarity requires a more detailed classification of behaviors. The examples that we present do not exhaust the possible behaviors, but each is "generic," in the mathematical sense that small changes in parameter values do not change the qualitative behavior of the system. This implies that the qualitative behavior of each example is typical of a whole class of systems.

\section{Equilibrium}

A mechanical system is at equilibrium if the forces acting on it are in balance. For example, when a body floats, the force of gravity is balanced by the buoyant force due to displacement of the liquid. The "balance of nature" (Pimm 1991 ) is an extension of this idea to the natural world. The concept usually refers to steady flows of energy and materials, rather than to a system whose components do not change.

\section{Resilience and stability}

We are interested in characterizing natural systems that are resilient, i.e., that tend to maintain their integrity when subject to disturbance (Holling 1973). This is related to the idea of stability. The informal concept of stability refers to the tendency of a system to return to a position of equilibrium when disturbed. If a weight is added suddenly to a raft floating on water, the usual response is for the weighted raft to oscillate, but the oscillations gradually decrease in amplitude as the energy of the oscillations is dissipated in waves and, eventually, in heat. The weighted raft will come to rest in a different position than the unweighted raft, but we think of the new configuration as essentially the same as the old one. The system is stable.

If we gradually increase the weight on the raft, eventually the configuration will change. If the weight is hung below the raft, the raft will sink deeper and deeper into the water as more and more displacement is required to balance the higher gravitational force. Eventually, the buoyant force cannot balance the gravitational force and the whole configuration sinks: the system is no longer stable. On the other hand, if the weight is placed on top of the raft, the raft may flip over suddenly and lose the weight and its other contents long before the point at which the system, as a whole, would sink. This sudden loss of stability may be more dangerous than the gradual sinking, because there may be little warning or opportunity to prepare for it. We may think of the raft system as losing its resilience as more weight is placed on it.

Suppose that we accept the "balance of nature" and the steady flows of resources that it implies. As we demand more and more of the products of natural systems, and we load them with more and more of our waste products, are we likely to experience a gradual loss of stability or a sudden one? In order to clarify such questions, we must refine our terminology. To decide whether a system is stable or not, we must first specify what we mean by a change in configuration or loss of integrity. If we don't care whether the raft flips over when weighted, then there is no problem of sudden loss of stability for the floating raft. We must also specify the types and quantities of disturbances that may affect the system. Suppose that a fixed weight is placed on top of an occupied raft. If the occupants of the raft move about, the raft may float at a slightly different angle, but if they move too far or all at once, the raft may tip. The range of possible movements of the occupants that do not lead to tipping is called the domain of stability, or domain of attraction, of the upright state. If the amount of the fixed weight is gradually increased, the balance becomes more precarious and, hence, the domain of attraction will shrink. Eventually, the weight becomes large enough so that there is no domain of attraction at all, and the raft will flip over no matter what its occupants do. 
The preceding example makes a distinction between the weight loading the raft and the positions of the occupants. If the amount of the weight changes very slowly or not at all, we may think of the "system" as consisting of the raft and weight. The occupants change position relatively quickly, and these changes may be thought of as disturbances of the system. On the other hand, we may adopt a more comprehensive point of view, seeing the raft, the weight, and the occupants as a single system. If the occupants organize themselves to anticipate and correct for external disturbances, then the system may be able to maintain its integrity long enough for them to achieve their objectives. Another possible response to disturbance might be to restructure the raft itself. If it were constructed of several loosely coupled subunits, then excessive weighting or a strong disturbance might flip one part of the system, but leave the rest intact. Such a structure might not require as much vigilance to maintain as the single raft, and it might be able to withstand a greater variety of external disturbances. On the other hand, if the bindings that link the subunits become stiff, then the structure may become brittle and, hence, more prone to failure. This simple example illustrates how the notion of resilience of a system depends upon our objectives, the time scale of interest, the character and magnitude of disturbances, the underlying structure of the system, and the sort of control measures that are feasible.

Section 2 presents the main ideas of stability and resilience for simple, one-dimensional prototype systems. Calculations can be done explicitly for these prototype systems, but their qualitative behavior holds for much more complicated examples. Section 3 illustrates the ideas of bistable equilibria, hard loss of stability, hysteresis, and resilience with a model for the spruce budworm. There are qualitative similarities between the behavior of the budworm model and a variety of ecological systems, particularly lake ecosystems, the Baltic Sea, and boreal forests, although no attempt is made here to provide a formal model for these systems. Such a model is given in Section 4 for a competitive grazing system. This system has qualitative behavior analogous to a one-dimensional model, and it also exhibits hysteresis and hard loss of stability. An analogous system that involves fire as a regulating process is presented in Section 5 . The latter system also exhibits regular oscillations. The Appendix presents a detailed account of the relationship between return times for a disturbed system and its resilience. There are two conflicting definitions of resilience, which may cause confusion. The definition of Pimm (1991) applies only to behavior of a linear system, or behavior of a nonlinear system in the immediate vicinity of a stable equilibrium where a linear approximation is valid. For Pimm, loss of resilience is due to slow dynamics near a stable equilibrium. The definition of Holling (1973) that we use here refers to behavior of a nonlinear system near the boundary of a domain of attraction. Loss of resilience, in our sense, is associated with slow dynamics in a region that separates domains of attraction.

\section{A SI MPLE PROTOTYPE FOR STABI LITY AND RESI LI ENCE}

In order to understand complicated systems, it is often convenient to consider a simpler system that exhibits the type of behavior of interest. A full theory of the floating raft would require a combination of the theories of hydrodynamics and of rigid body dynamics, but the essential features can be captured in a one-dimensional model. We are mainly concerned with the notion of stability and the fact that the domain of attraction of a stable equilibrium may depend upon slowly varying parameters. These features are present in a one-dimensional system.

\section{Global stability}

The concept of the balance of nature might be taken to imply that the system will maintain its integrity under any sort of perturbation. Such an assumption may be made (often unconsciously) when we make large modifications to natural systems. Our expectation is that things will proceed more or less as before, and that the response of the system will be approximately proportional to the perturbation. Such behavior is shown by the simplest linear models. Some might argue that a principle of parsimony dictates that such models be used in the absence of strong evidence to the contrary. The following linear model illustrates the property of global stability, which implies that the system will always return to a certain equilibrium, regardless of how far it is displaced from that equilibrium. 


$$
\frac{d \tau}{d t}=h(\alpha)-\tau
$$

where $h(\alpha)$ is a smoothly varying function of an external variable $\boldsymbol{\alpha}$ and $\mathbb{E}$ is the quantity of interest. Then $d \tau / d t=0_{\text {if }} \tau=h(\alpha)$; the system has a single equilibrium there. This equilibrium is stable, since $d \tau / d t>0$ if $x<h(\alpha)$, and $d x / d t<0_{\text {if }} \boldsymbol{x}>h(\alpha)$. These relations imply that the system approaches the equilibrium, no matter what the starting point.

A system such as (Eq. 1) cannot fail us or surprise us. It returns to an equilibrium, no matter how far it is displaced, and the position of the equilibrium changes smoothly with the exogenous variable $\alpha$. Such a system is not suitable for a discussion of possible collapses of natural systems, since such collapses are excluded by assumptions such as (Eq. 1). Mathematical theory provides numerous examples of different behavior, and our goal is to investigate their plausibility. Unfortunately much theory (including most economic theory) has been based upon assumptions analogous to (Eq. I). In particular, "resilience" has been defined by Pimm (1991) in terms of the system (Eq. 1), and this very special assumption may mislead the unwary. There is a mathematical theory that shows that systems such as (1) are good approximations to general systems with a stable equilibrium, but that theory implies that the approximation holds only in the immediate vicinity of the equilibrium, i.e., that the approximation is valid only locally. Details are provided in the Appendix.

\section{Bifurcation}

In order to explore the differences between local and global stability, we must examine nonlinear models, i.e., models in which the state variable appears in more complicated functions than linear ones. The following example has three equilibria instead of a single one. Such a complication requires a cubic or more compliocated dependence upon the $\boldsymbol{T}$ variable, for example,

$$
\frac{d x}{d t}=f(x)=x\left(x^{2}-\alpha\right)
$$

Here, $\alpha$ is a parameter or a slowly varying quantity whose dynamics are not of immediate concern. The equilibria of the system are the states where $f(x)=0$. These are the states where either of the two factors in (Eq. 2 ) vanishes. Hence, they are points where

$$
x=0 \text { or } x^{2}=\alpha \text {. }
$$

If $\alpha>0$, then there are three equilibria

$$
x=0, \quad x=\sqrt{\alpha} \text { or } x=-\sqrt{\alpha} \text {, }
$$

but if $\alpha \leq 0$, then there is only the single equilibrium at $\tau=0$. Such a change in the configuration or stability of equilibria is a called a "bifurcation" (Guckenheimer and Holmes 1983). It implies a change in the qualitative behavior of the system. To explore this feature, we must discuss some additional concepts.

\section{Local stability and domain of attraction}

In order to determine the stability of equilibria, it suffices to examine the sign of the velocity of $\boldsymbol{T}$. For example, if $\alpha<0$, the second factor in (Eq. 2 ) is always positive and, hence, $d \tau / d t>00_{\text {if }} \boldsymbol{\tau}>0$ and $d \tau / d t<0$ if $\mathbb{I}<0$. In this case, the system always moves away from the state where $\mathbb{I}=0$. We conclude that the equilibrium at $\mathbb{I}=0$ is unstable if $\alpha<0$. On the other hand, if $\alpha>0$, then $d \tau / d t$ changes sign at three places: 


$$
\begin{aligned}
& \frac{d x}{d t}>0 \text { if } x>\sqrt{\alpha}, \\
& \frac{d x}{d t}<0 \text { if } 0<x<\sqrt{\alpha,} \\
& \frac{d x}{d t}>0 \text { if }-\sqrt{\alpha}<x<0, \\
& \frac{d x}{d t}<0 \text { if } x<-\sqrt{\alpha},
\end{aligned}
$$

The equilibrium where $\mathbb{I}=\sqrt{\alpha}$ is unstable, because the system always moves away from that point if nearby (according to Eqs. 5 and $\underline{6}$ ). Similarly, the equilibrium where $\bar{x}=-\sqrt{\alpha}$ is unstable (according to Eqs. 7 and $\underline{8}$ ). On the other hand, the equilibrium where $\boldsymbol{I}=0$ is stable (according to Eqs. 6 and 7), because the motion from nearby points is toward that point. However, if the system starts outside the interval $(-\sqrt{\alpha}<\tau<\sqrt{\alpha})$, it moves away from the equilibrium at $\mathbb{I}=0$. Therefore, the equilibrium at $\mathbb{I}=0$ is locally stable, but not globally stable. The system returns to $\mathbb{I}=\mathbb{0}$ if small perturbations are made, but larger perturbations take the system into an unstable domain. The interval $(-\sqrt{\alpha}<\pi<\sqrt{\alpha})$ is called the "domain of attraction" of the point $\pi=0$, because trajectories that start within that interval eventually return to $\mathbb{I}=0$, but not those that start outside.

It is clear that the domain of attraction of the stable equilibrium at $\boldsymbol{I}=0$ shrinks as $\boldsymbol{\alpha}$ decreases toward zero. The three equilibria collapse into one where $\alpha=0$, and only a single unstable equilibrium remains when $\alpha<0$. This information is summarized in Fig. 1. The diagram looks like a branch and, for this reason, it is called a

" bifurcation diagram." The domain of attraction of the point $\mathbb{I}=0$ is contained within the two curved branches, and there is no other domain of attraction.

FI G. 1. The parameter $\alpha$ is plotted on the horizontal axis and the corresponding equilibria in $\mathbb{I}$ for $\mathrm{Eq}$. (2) are plotted on the vertical axis. The stable equilibrium is plotted with a solid curve, whereas unstable ones are plotted with dotted curves. 


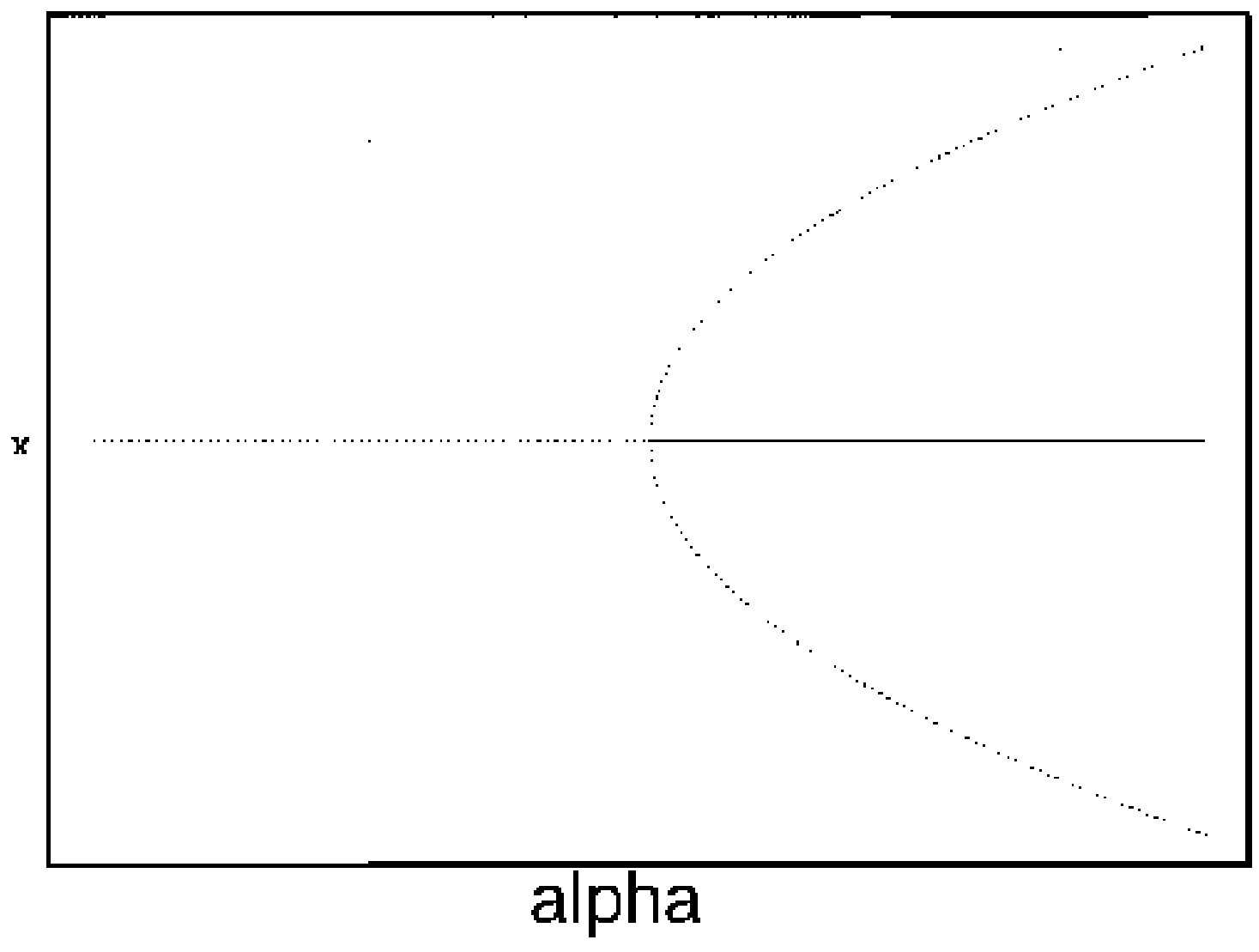

Increasing evidence has accumulated for the existence of multistable states in nature: coral reefs (Done 1992, Hughes 1994, McClanahan et al. 1996), African rangelands (Dublin et al. 1990), shallow lakes (Schindler 1990, Carpenter and Leavitt 1991, Scheffer et al. 1993), kelp forests (Estes and Duggins 1995), and grasslands (D'Antonio and Vitousek 1992, Zimov et al. 1995).

\section{Disturbances and slow parameter changes}

We have seen that if $\alpha>0$, then this system approaches the stable equilibrium at $\mathbb{I}=0$ if it is started within the domain of attraction. If we envisage disturbances that displace the system a distance $\mathbb{I}_{\mathbf{l}}$ from the stable equilibrium, they will not affect the integrity of the system (its tendency to return to the 0 state) as long as $x_{1}^{2}<\alpha$. Now, if we allow the parameter $\alpha$ to decrease slowly toward $x_{1}^{2}$, the system will take longer and longer to return to the state $\mathbb{I}=\mathbb{0}$ when $\mathbb{I}$ is displaced to $\mathbb{I}_{\mathbf{l}}$, because motion is very slow near $\mathbb{I}=\sqrt{\boldsymbol{\alpha}}$, and a disturbance of magnitude $\mathbb{L}_{1}$ may take the system into the region of slow dynamics. We may think of the decrease in $\alpha$ as causing a loss of resilience, because the integrity of the system is threatened more and more by disturbances of a given magnitude. A symptom of loss of resilience may be longer and longer times to return to the vicinity of $\boldsymbol{I}=0 \mathrm{0}$ after disturbance. The connection between return times and resilience is not completely straightforward; we address it in some detail in the Appendix.

\section{Two domains of attraction}

The preceding system is not a believable model for natural systems, because it predicts that the state variable may approach infinity under some circumstances. A more plausible scenario is one in which the system may change from having a single stable equilibrium to one with two stable equilibria. We next consider a number of such "' bistable" systems. 
We obtain a simple prototype for such systems by changing the sign of $d x / d t$ in (Eq. 2). If the direction of time is reversed, the stable and unstable equilibria are interchanged. If $\alpha<0$, then the single equilibrium at $\boldsymbol{I}=0$ is globally stable: the system always returns to that equilibrium no matter where it starts. Instead of two unstable equilibria when $\alpha>0$ (as in the former case), there will be two stable equilibria. The new system is

$$
\frac{d x}{d t}=-f(x)=-x\left(x^{2}-\alpha\right) \text {. }
$$

If $\alpha>0$, for this system, we have

$$
\begin{aligned}
& \frac{d x}{d t}<0 \text { if } x>\sqrt{\alpha}, \\
& \frac{d x}{d t}>0 \text { if } 0<x<\sqrt{\alpha}, \\
& \frac{d x}{d t}<0 \text { if }-\sqrt{\alpha}<x<0, \\
& \frac{d x}{d t}>0 \text { if } x<-\sqrt{\alpha},
\end{aligned}
$$

If $\mathbb{I}>\mathbb{0}$ initially, then $\mathbb{I}$ heads toward the equilibrium at $\mathbb{I}=\sqrt{\boldsymbol{\alpha}}$, but if $\mathbb{I}<0$ initially, then $\mathbb{I}$ heads toward the equilibrium at $\mathbb{I}=-\sqrt{\alpha}$. Thus, the domain of attraction of the point $\mathbb{I}=\sqrt{\alpha}$ is the positive $\mathbb{I}$ axis, and the domain of attraction of $\boldsymbol{I}=-\sqrt{\boldsymbol{\alpha}}$ is the negative $\boldsymbol{I}$ axis. Each of the stable equilibria is locally stable, but not globally stable. This system can be flipped from one stable state to another by crossing the unstable line where $\boldsymbol{I}=\mathbb{0}$. Because this line separates the two domains of attraction, it is called a " separatrix." The equilibria in $\mathbb{I}$ are plotted in Fig. 2 .

FI G. 2. Equilibria in $\boldsymbol{T}$ for $\mathrm{Eq}$. (9) are plotted vs. $\boldsymbol{k}$, as in Fig. 1 . The direction of increase of time is reversed as compared with Fig. 1; hence, the stable and unstable equilibria are interchanged. 


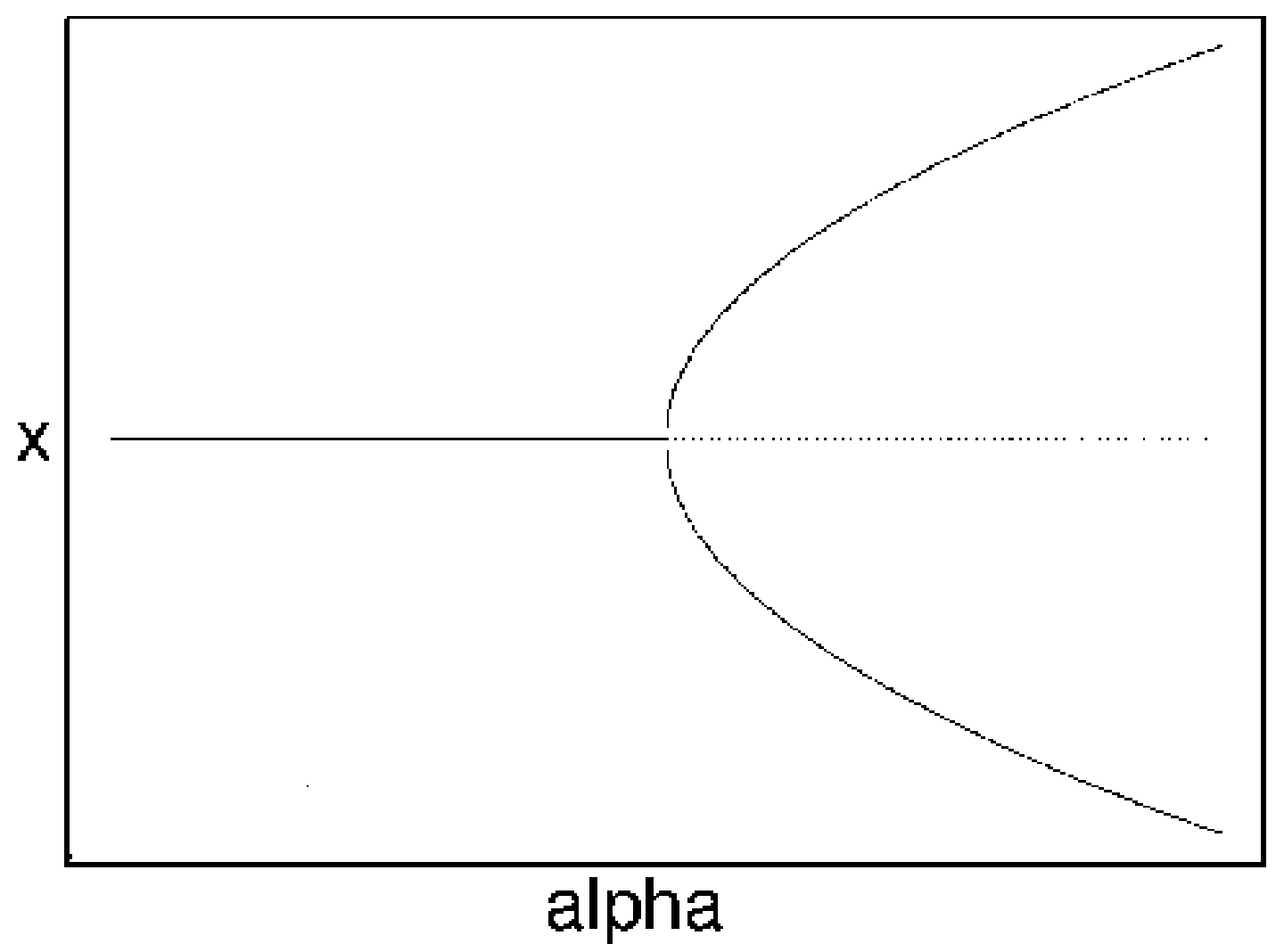

\section{Disturbances and slow parameter change}

The bifurcation diagram in Fig. 2 implies a great deal about the response of the system to disturbance. If $\alpha<<$, then the system will return to the stable equilibrium at $\boldsymbol{I}=[\mathbf{0}$, no matter how large the disturbance: there is nowhere else for it to go. However, if $\alpha>0$ and the system starts near the lower branch, it will tend to return there if displaced by a small amount. As $\alpha$ decreases toward zero, the distance between the stable equilibria and the unstable one decreases. Hence, disturbances of a given magnitude take the system closer and closer to the unstable equilibrium. Dynamics are slow near the unstable equilibrium and, hence, the time to return to the vicinity of the lower branch increases sharply for trajectories that approach the unstable equilibrium. This point about return times can be made precise by a calculation analogous to that in the Appendix.

For a higher level of disturbance and $\boldsymbol{\alpha}>0$, , the system may be moved across the separatrix at $\mathbb{I}=0$, and may approach the upper branch of stable equilibria. Under a random pattern of disturbances, we may expect to see the system spend long periods of time in the vicinity of one or the other of the stable equilibria. Every now and then, the random disturbances may combine and send the system to the other stable equilibrium. The "'Allee effect"' studied by ecologists provides an example of a bistable system. A population may suffer reduced survival or reproductive success at low numbers. For example, schooling fishes tend to suffer low per capita mortality if their numbers are high enough relative to the capacity of their predators. If such fish are reduced in numbers through fishing pressure or environmental degradation, the population may decline and eventually become extinct locally. On the other hand, a large population may sustain itself over long periods. Dynamics of this sort might explain the occasional flips between dominance of sardine and anchovy, as revealed by deposits of their scales off the coast of California. A similar pattern appears in such geophysical features as the polarity of the earth's magnetic field, the ocean circulation involving the Gulf Stream, and climate fluctuations, but some of these fluctuations may be too regular to be completely random. For larger values of $\alpha$, one would expect flips from one equilibrium to the other to be extremely rare. Thus, we may associate an increase in $\alpha$ with an increase in resilience.

\section{HARD LOSS OF STABI LITY AND HYSTERESIS}


The two preceding examples illustrate a so-called " soft loss of stability." As the exogenous variable changes, the location of the stable equilibria changes smoothly. The state variable may move from one domain of attraction to another, but such changes are slow because dynamics are slow near an unstable equilibrium or a separatrix. The possibility of such behavior would not ordinarily be cause for alarm, because slow dynamics may allow for adjustments to new behavior. There are natural systems, such as outbreaking insect populations, that sometimes show more abrupt changes.

The following model was used by Ludwig, Jones, and Holling (1978) to understand the dynamics of the spruce budworm. The quantity $B$ represents budworm density, measured in larvae per acre. This density is assumed to vary in time according to

$\frac{d B}{d t}=r_{B} B\left(1-\frac{B}{K_{B}}\right)-\beta \frac{B^{2}}{\alpha^{2}+B^{2}}$

where $r_{B}$ is an intrinsic growth rate at low densities, $K_{B}$ is a carrying capacity for the budworm in the absence of predation, and the second term in (Eq. 14) is a predation rate. The predators are assumed to have a Holling type-

III functional response, with a maximum predation rate of $\beta$ and a half-saturation budworm density of $\alpha$. This functional form implies that predators have their greatest influence upon dynamics at intermediate ranges of budworm densities. At low densities, the predators search for alternate prey, because returns from foraging for budworm are relatively low. At high densities, budworms swamp their predators; thus, the predators have a small per capita effect, just as predators have a small per capita effect on large schools of fish. The parameter $\boldsymbol{\alpha}$ is proportional to a measure of foliage density, because the predators search foliage for the budworms and their response is mediated by the number of budworms per unit of foliage. Hence, $\boldsymbol{\alpha}$ is actually a state variable that generally changes on a slower time scale than the budworm. For the moment, we regard $\alpha$ as a constant.

Some algebra supplied in Ludwig et al. (1978) shows that there are either two or four equilibria for the budworm, depending upon the sizes of the dimensionless parameters $R$, and $Q$, given by

$$
R=\frac{r_{B}^{\alpha}}{\beta}, \quad Q=\frac{K_{B}}{\alpha} .
$$

These equilibria satisfy

$$
R b\left(1-\frac{b}{q}\right)-\frac{b^{2}}{1+b^{2}}=0
$$

where $b=B / \alpha$. The equilibrium $b=0$ is always unstable, because $d b / d t>0$ highest equilibrium is always stable, because $d b / d t<0$ if $\boldsymbol{b}$ is very large and positive. Thus, if there are only two equilibria, budworm density always moves toward the upper equilibrium. When there are four equilibria, they alternate in stability. A typical case is shown in Fig. $\underline{3}$. If $R_{\text {, is between }} R_{\mathbf{l}}$ and $R_{\mathbf{2}}, \boldsymbol{b}$ may approach either the high equilibrium or the low equilibrium, depending upon whether the starting position of $\boldsymbol{b}$ is above or below the unstable equilibrium, which is the separatrix.

FI G. 3. The equilibria in $\boldsymbol{b}$ for Eq. (16) are plotted against $R$, for $Q=20$. The points $R_{\mathbf{l}}$ and $R_{2}$ delimit the interval on the $R$, axis where there are three nonzero equilibria for the system. 


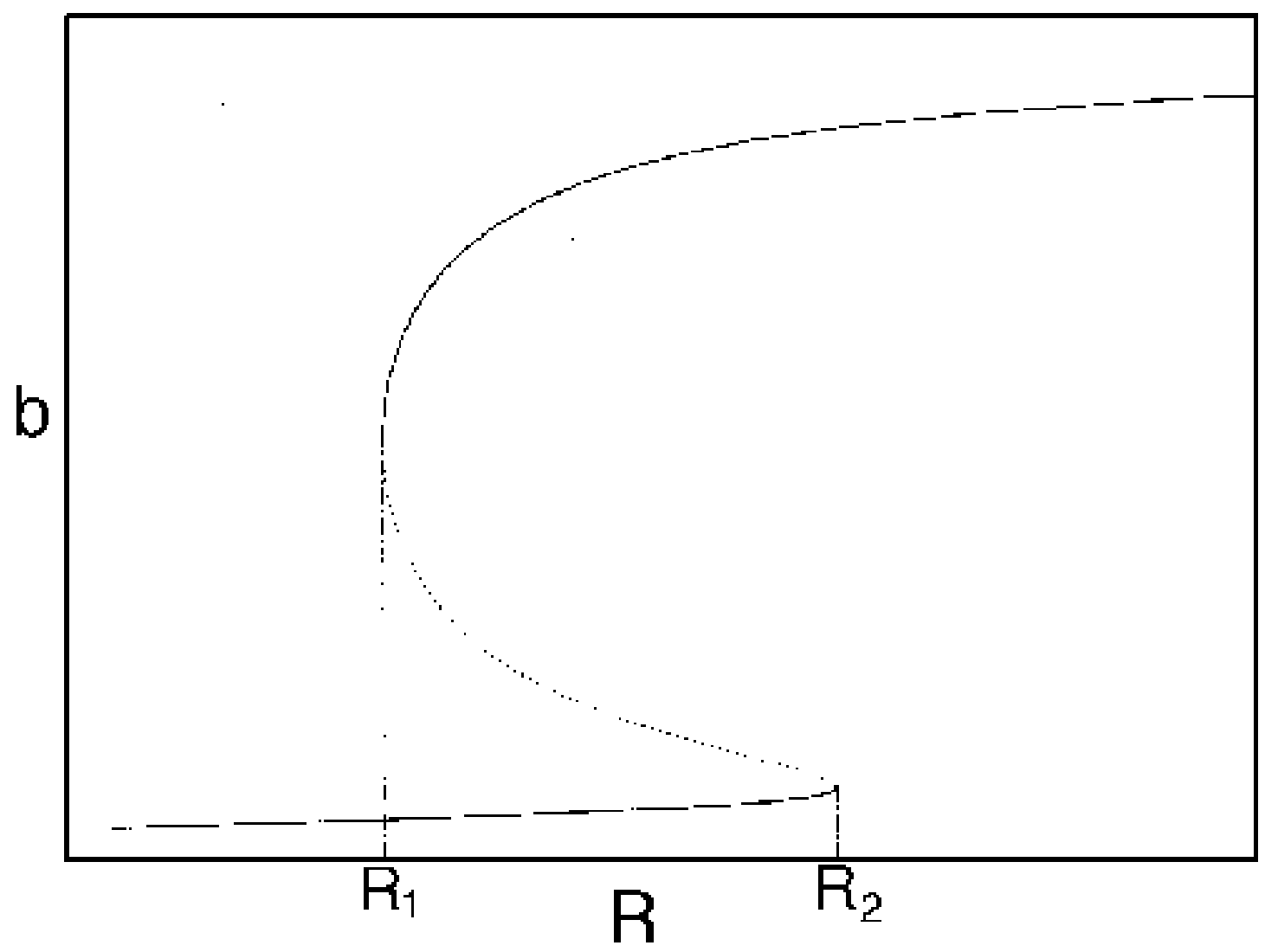

\section{Hard loss of stability}

Imagine that the parameter $\alpha$ begins at a low value and gradually increases as the forest grows. It turns out that $Q$ does not change with forest growth; hence, Fig. 3 applies. Because $R$, is proportional to $\alpha, R$, will increase. At first (when $R<R_{1}$ ), budworm numbers will remain low, as the only stable equilibrium is the low one. Even when $R$ increases beyond $R_{1}$, the budworm numbers will remain low, because they lie below the unstable equilibrium, which determines the domain of attraction of the low equilibrium. The stability of the low equilibrium becomes precarious as $R$, approaches $R_{\mathrm{Z}}$, because the domain of attraction shrinks. Finally, at $R=R_{\mathrm{Z}}$, the lower two equilibria disappear and budworm density jumps to the high value: an outbreak occurs. This abrupt change in the attracting state is called a "hard loss of stability." It should be contrasted with the soft loss of stability displayed by the system in ( $\mathrm{Eq} .9$ ). In the case of the budworm, once density has reached the high equilibrium, there is no easy way to reduce it to the lower equilibrium. If the variable $R$ is reduced below $R_{2}$, the budworm remains at the high equilibrium. As $R$, is further reduced, there is a second hard loss of stability as $R$, declines below $R_{1}$. In this case, there is a jump down to the low equilibrium, which is not reversed as $R$, increases again.

\section{Hysteresis and cycles}

If we now connect the dynamics of the trees and the dynamics of the budworm, a new phenomenon appears. If the system starts with low foliage density and low budworm numbers, the foliage density slowly increases until it surpasses $R_{2}$. At this point, an outbreak occurs, as shown previously. High budworm numbers eventually cause death of trees, so $R$, begins to decrease when the budworm has an outbreak. Budworm numbers remain high even though $R$, declines, because budworm density lies above the separatrix. As $R$, continues to decline to $R_{1}$, budworm density declines slowly and then jumps to a low value when $R$, decreases below $R_{1}$. The different paths followed by 
the total system for increasing vs. decreasing $R$, constitute the "hysteresis effect." The combination of budworm and forest dynamics produces stable cycles with long periods. Such stable cycles that are maintained through alternations of rapid transions and slow changes are called " relaxation oscillations." They are common in many physical, chemical, and physiological systems (Edelstein-Keshet 1988).

\section{Disturbances and resilience}

If the objective of management is to keep budworm numbers and foliage damage low, the loss of stability as $R$, increases beyond $R_{2}$ may be regarded as a loss of resilience. This model suggests that small disturbances near the lower stable equilibrium may exhibit long return times if they approach the unstable branch. Such long return times may be a useful diagnostic indicator. However, because $R$, increases as trees grow, a loss of stability accompanied by a budworm outbreak seems inevitable.

We may adopt a different perspective and regad periodic budworm outbreaks as part of a stable system that renews the forest from time to time. Indeed, systems analogous to the budworm-forest system frequently appear as stable oscillators. The advantage of such oscillators is that they continue to oscillate more or less with the same frequency and amplitude under a wide variety of disturbances. Hence, physiological oscillators are important in maintaining integrity of the organism, which is another kind of resilience. According to this perspective, an attempt to halt the oscillations may lead to a disastrous breakdown in the long term. Will human interventions to increase productivity in natural systems suffer a similar fate?

\section{Lake dynamics}

Carpenter et al. (1996) have discussed the applicability of these ideas to lake ecosystems. They characterize lake dynamics as either "normal" or "pathological." Normal lakes have high numbers of game fish, effective grazing upon phytoplankton, and low incidence of algal blooms. The normal system maintains its integrity when subjected to perturbations such as phosphorus pulses, because phosphorus moves rapidly into the higher trophic levels (Carpenter and Kitchell 1993) and humics constrain algal growth (J ones 1992). However, heavy phosphate loading, removal of macrophytes, overfishing, and removal of wetlands and riparian vegetation may lead to the pathological state in which there are few game fish, less grazing, no macrophytes, and extensive and frequent algal blooms (Harper 1992). This may be a rapid transition and it is not easily reversed (National Research Council 1992).

This situation appears to fit the definition of a hard loss of stability, because the change is rapid and large, and is sometimes not reversed even if phosphate loads are decreased (National Research Council 1992). One may say that the normal lake is resilient because it maintains its integrity under perturbation, but resilience is lost as phosphate loading and other stresses are increased. If critical levels of phosphate and other environmental variables could be identified, we might attempt to measure resilience in terms of the difference from the critical levels (Vollenweider 1976). Perhaps the question of whether or not the lake ecosystem fits our definitions may be answered by statistical analysis of long-term data.

\section{The Baltic Sea}

Jansson and Velner (1995) describe the Baltic system in terms that show many similarities to the lake system. The Baltic Sea is partially enclosed and, consequently, has a residence time of water on the order of 20 years. Algae form the base of a diverse food web, with higher trophic levels occupied by commercially important species such as herring, flounder, pike, and perch. There may be long periods when there is weak vertical mixing of the water column, due to lack of inflow from the North Sea. During such periods, oxygen levels at greater depths may be very low and sulphur bacteria may predominate. The latter put large quantities of phosphorus into solution, which then upwell and cause plankton blooms. 
In historical times, the Baltic has experienced several extended anoxic periods, but the system has not been permanently altered. Since the industrial revolution, the Baltic has been loaded with increasing amounts of phosphorus, and there are indications of a change of configuration to a detritus-based system. This would imply more turbid water and a fish community consisting mainly of sluggish species such as bream, roach, and ruffe, which are much less valuable than those previously listed. The possibility exists that the Baltic might reach a point at which even reducing phosphate inputs might not return the system to its earlier, more desirable state. Such a turn of events would correspond to a hard loss of stability, analogous to the behavior of the lake ecosystem. Unfortunately, there are no replicates of the Baltic system; hence, we have only analogies to guide action. A purposeful demonstration that the Baltic is actually capable of a sudden change corresponding to a hard loss of stability is unthinkable as an experiment. Nevertheless, it may be occurring as a result of human negligence. The earlier ability of the Baltic to recover from anoxic periods may correspond to resilience, but we cannot be sure whether this resilience is being lost.

\section{The boreal forest}

Carpenter et al. (1996) characterize the boreal forest as a system with relatively few species and complicated interactions and dynamics. In upland regions, forests dominated by aspen and birch alternate with forests dominated by spruce and fir. Browsing by moose over a period of 20-40 years can convert an aspen stand into one dominated by conifers. As stands of conifers mature, they become increasingly favorable for reproduction of the spruce budworm. Eventually, outbreaks occur and portions of the system are converted into early successional aspen. The budworm outbreak corresponds to a hard loss of stability, and the combined upland system undergoes stable, long-period oscillations analogous to those described previously.

As the upland regions undergo these oscillations, the valley bottoms alternate between flooded plains and moist meadows. The flooded state is maintained by beavers, which cut aspen bordering streams for food and dam the streams to create ponds. When the supply of aspen is insufficient, the beavers abandon their dams, the dams break, and the ponds are soon replaced by meadows. This relatively rapid change, a consequence of decreasing supply of aspen, may be thought of as a hard loss of stability. The upland and lowland cycles tend to entrain each other because of the interaction between beavers and aspen. Fires also play a role in synchronizing cycles over large spatial areas, because conifers killed by the spruce budworm provide an abundance of fuel.

Although this system undergoes large alterations, sometimes very quickly, it may be thought of as resilient, maintaining its character over many centuries. Conditions at any given site may change abruptly, but the system is usually a mosaic of patches at differing stages of the cycle. When considered as a whole, it maintains considerable diversity.

\section{A COMPETITI VE GRAZI NG SYSTEM}

In this section, we describe a natural system that may be bistable. Competition between grasses and woody vegetation in a semiarid environment is described in Walker et al. (1981). Suppose that either the grass or the woody vegetation has an advantage when at high densities relative to the other. In such a case, the system has stable equilibria that correspond to high levels of grass and woody vegetation, respectively. The competition is also influenced by the stocking rate of cattle, which consume grass but not woody vegetation. We shall regard the two plant forms as the dynamic state variables, and the stocking rate as a slowly varying parameter.

I magine starting with high levels of grass and low levels of woody vegetation. At low levels of stocking, there is only a small difference from the ungrazed system: if the system starts out with grass dominant, grass will continue to dominate. As stocking increases, the competition may favor woody vegetation. Eventually, there may be a collapse of the grass, and woody vegetation will dominate. Thus, the effect of grazing is to move the system from a state in which grass dominates to one in which woody vegetation dominates. Even when grazing pressure is relaxed, there may be little change in composition, because of the advantage enjoyed by woody vegetation over grass when the former is dominant. The effect of grazing is to move the system into the domain of attraction of woody vegetation for the ungrazed system. 
If one plots grass density vs. the stocking level, the behavior may appear to be inexplicable: the grass level declines as grazing increases, but does not return to former levels when grazing returns to its former level. The apparent paradox is resolved if we realize that the density of grass depends not only on the stocking level, but also on competition with woody vegetation. These phenomena may be illustrated by a modification of the Lotka-Volterra competition model.

\section{Mathematical model}

Let $G$ represent the density of grass, and let $w$ represent the density of woody vegetation. The rate of change of grass density is assumed to be

$$
\frac{d g}{d t}=r_{g} g\left(1-s-c_{g a} g-c_{w g} w\right) \text {, }
$$

where $r_{S}$ is a growth rate, and $C_{g}$ and $C_{w}$ are competition coefficients. The parameter $s$ is determined by the stocking rate of cattle. The rate of increase of the woody vegetation is assumed to be

$d w$

$\frac{d w}{d t}=r_{w}\left[a+w\left(1-c_{b w g}-c_{w w} w\right)\right]$

where $r_{w}$ is a growth rate, $C_{g w}$ and $C_{w w}$ are competition coefficients, and $\boldsymbol{a}$ is a source term. In the illustrations that follow, the parameters were chosen as:

$r_{w}=1, r_{g}=1.5, c_{g j}=.7, c_{w g}=1, c_{g w}=2, a=.03, c_{w w}=1+a$.

The case of light grazing corresponds to $s=1 / 10$.

In order to understand the behavior of this system, it is helpful to plot some curves in the $g, w$ plane, as in Fig. 4. In the phase plane, the direction and speed of change of the system are given by the vector $(d g / d t, d w / d t)$. This vector is vertical on the curve where $d g / d t=0$ (the null $G$ isocline), and it is horizontal on the curve where $d w / d t=0$ (the null $w$ isocline). As can be seen, $d g / d t=0$, where either $g=0$, or

$$
c_{g g} g+c_{w g} w=1-s \text {. (20) }
$$

This locus is a straight line, and it shifts to the left as $\mathbf{s}$ increases. The null $w$-isocline is a hyperbola according to (Eq. 18). One of the asymptotes is the $w$-axis, and the locus passes through the point $g=0$, $w=1$, labelled "W." This locus is independent of the stocking parameter $\mathbf{S}$. Figure 4 shows in detail how a system may approach more than one steady state, depending upon the starting conditions. Although this system is much more complicated than ( $\mathrm{Eq} .9$ ), its qualitative behavior is the same if $\alpha>0$. This illustrates how the very simple onedimensional models may, nevertheless, be a valuable heuristic guide.

FI G. 4. The phase plane for grass and trees, according to Eq. (17), Eq. (18), and Eq. (19). The null 9 and $w$ isoclines are plotted with dashed lines. These are the curves where $d g / d t=0$ or $d w / d t=0$, respectively. The equilibria are points where the isoclines cross. The stable equilibria are labelled as "G" and "W," respectively. The unstable equilibrium is labelled as "S." The trajectories that enter $\mathrm{S}$ form a separatrix: they are plotted with dotted lines. Trajectories to the right of the separatrix eventually reach G, and those to the left eventually reach $\mathrm{W}$. 


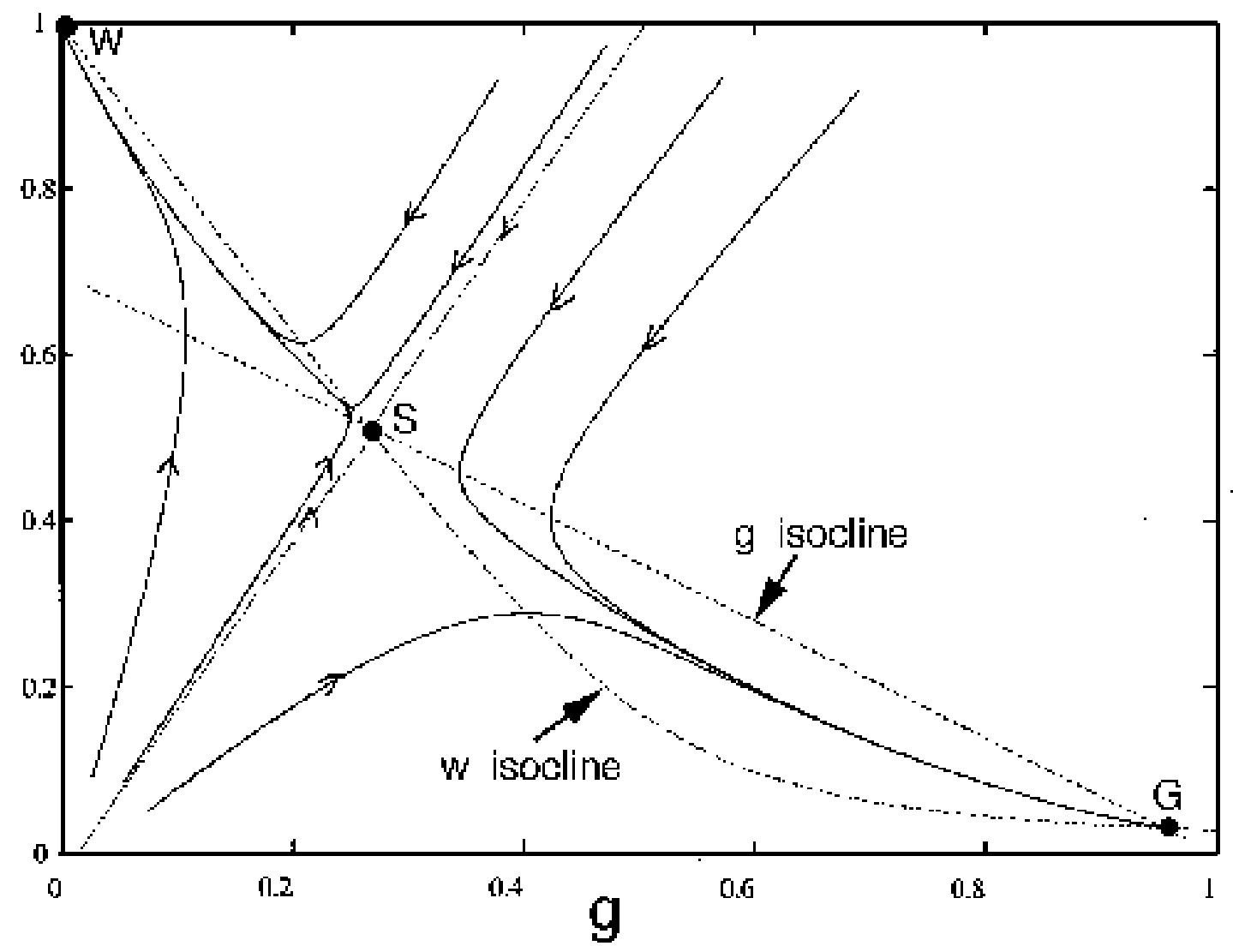

We now turn to the effect of increased stocking. The effect of an increase in $\mathbf{S}$ is to shift the null 9 -isocline down and to the left. Hence, the points $S$ and $G$ will approach each other along the $w$ null isocline. Because the separatrix passes through the point $\mathrm{S}$, the domain of attraction of $\mathrm{G}$ must shrink, whereas the domain of attraction of W will expand. Qualitatively, the representation of Fig. 4 still holds. For a still higher value, $\mathbf{s}=\mathbf{s}^{*}$, the points $\mathbf{S}$ and $\mathrm{G}$ coincide. The values of $G$ corresponding to the roots $S$ and $G$ are shown in Fig. 5 . A similar diagram could be drawn to show the corresponding values of $w$. This is a bifurcation diagram analogous to Fig. 3 . If the stocking rate changes slowly, we may expect the grass density to be given by the upper curve in Fig. 5 . However, if $s^{>} s^{*}$, the grass density must crash, since there is no stable equilibrium with a nonzero grass density. This is a hard loss of stability, and indeed there is a striking similarity between Figs. 5 and $\underline{3}$.

FI G. 5. A bifurcation diagram showing grass equilibria as a function of the grazing parameter $\mathbf{s}$ for the system (17)-(19). If $s<s^{*}=.4848$, there are three roots. The lowest branch corresponds to the point $W$ where $g=0, w=1$, which is always a stable equilibrium. The middle branch corresponds to the locus of point $\mathbf{S}$ as $\mathbf{s}$ varies. Those points are unstable equilibria, so they are plotted with a dotted line. The highest branch is the locus of point $\mathbf{G}$ as $\mathbf{s}$ varies. This point is always stable. 


\section{Grass equilibria vs grazing}

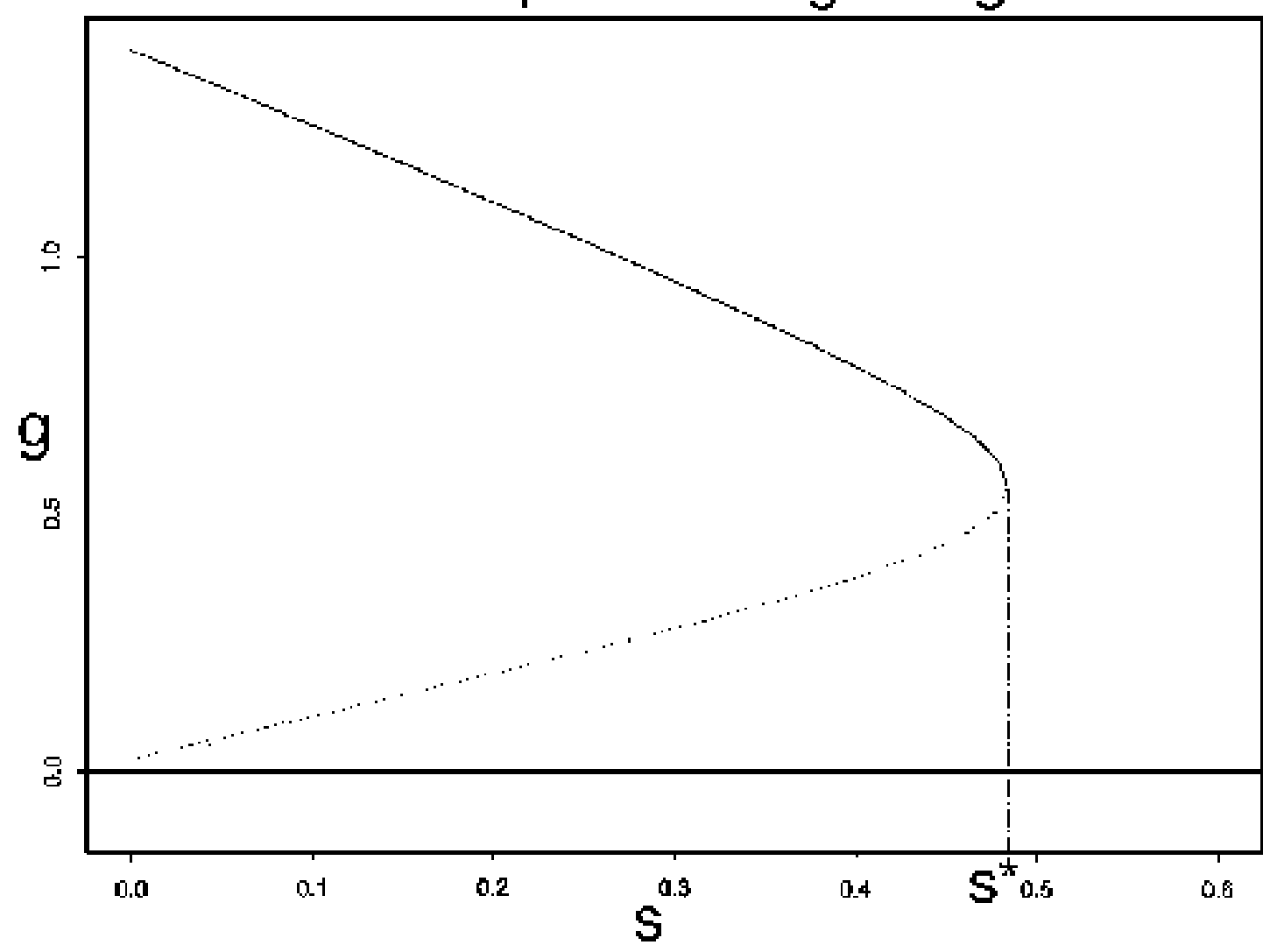

For values of $\mathbf{s}>\mathbf{s}^{*}$, the qualitative form of Fig. 6 applies. The only equilibrium is point W, and all trajectories approach $\mathrm{W}$. The domain of attraction of $\mathrm{W}$ is the whole quadrant where $g>0$ and $w>0$.

FI G. 6. The phase plane for grass and trees for the system (17)-(19), with $s=.6$. In this case, there is only a single stable equilibrium for the system at $g=0, w=1$. All trajectories approach that point. 


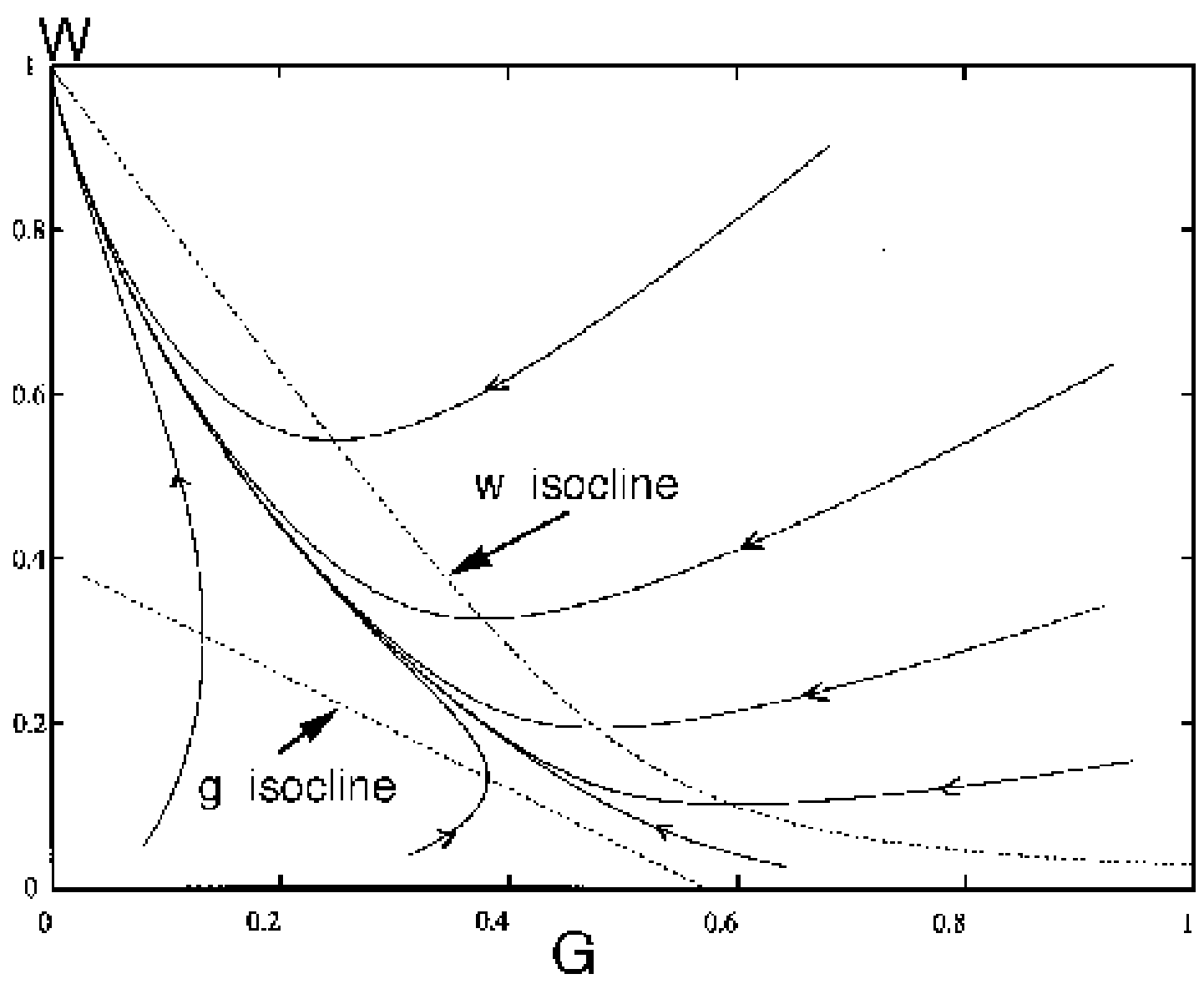

We may imagine the system beginning with the stocking rate $\mathbf{s}=0$. According to Fig. 5 , the unstable equilibrium $\mathrm{S}$ and the stable equilibrium $\mathrm{W}$ are very close together. Hence, the separatrix in a figure analogous to Fig. 4 is in the extreme upper left corner: virtually any initial combination of $G$ and $w$ will lead to a high density of 9 , given by the upper branch in Fig. 5 , with a correspondingly small density of $\boldsymbol{W}$. Now, if $\mathbf{s}$ is increased slowly, the density of $G$ will move downward along the upper branch until the bifurcation point $\mathbf{s}^{*}$ is reached. Beyond that point, the grass density must crash, and woody plants will dominate. Even if grazing pressure is withdrawn ( $\mathbf{S}=$ (0), the grass cannot recover because it will be to the left of the separatrix. This is the hysteresis effect.

\section{FI RE I N A SAVANNA SYSTEM}

For two reasons, the preceding model does not describe most savanna systems. (1) Generally, neither grass nor woody vegetation can completely exclude the other. Instead, there is a single stable equilibrium for the system (which changes over time, depending upon rainfall, grazing, and fire) where grass and trees coexist. (2) Woody vegetation cannot exclude grass indefinitely: after a long period of low grazing, the grass may return. This is due to a combination of two effects. First, the older woody vegetation may die and leave gaps that may be colonized by grass, and then fire gets into the system. Second, woody vegetation dies back very quickly in dry years, but recovers only slowly in wet years, too slowly to use all the water. Grass, on the other hand, can increase 10-fold in a season, quickly enough to fully use all of the available water. Because of this, the combination of wet and dry years keeps woody vegetation at lower levels than the average rain would sustain, and permits grass to remain in the system in significant amounts.

When viewed at a long time scale, a brief period of grazing may cause a rapid collapse of the grass, followed by a slow recovery. What appears to be an equilibrium with high woody vegetation, when viewed at a short time scale, corresponds to a region in which the system spends a long time when viewed at a long time scale. This sort of qualitative behavior is analogous to "excitable systems." Such systems are best known as models of the nerve impulse, according to the theory of Hodgkin and Huxley, as modified by Fitzhugh. The system has a single stable equilibrium, but when perturbed in an appropriate direction, it may undergo a very large excursion (firing of the 
neuron), followed by a long recovery (refractory) period. Details are given in Edelstein-Keshet (1988).

\section{The effect of fire on dynamics}

To model aging properly is complicated, but for present purposes, it suffices to find a simple system that has the required qualitative behavior. We do not contend that the following model is an accurate representation of the true dynamics. We must keep track of surplus grass that may serve as fuel for fires. Hence, we let gross grass production be given by $\Phi_{p}$, where

$$
g_{p}=r_{g} g+a_{g}
$$

where $a_{g}$ is a source term, and $r_{g}$ is the rate of grass production available for grazing. The grass that is not consumed by grazing cattle is potential fuel for fires, and is denoted by $g_{f}$, where

$$
g_{f}=g_{p} \exp (-s g) \text {. }
$$

The parameter $\mathbf{S}$ determines the proportion of grass consumed by cattle. Now (Eq.17) is replaced by

$$
\frac{d g}{d t}=g_{f}-g_{p}\left(c_{g b g}+c_{w_{g}} w\right)
$$

The dynamics of $w$ will be influenced by fires, and the age of trees influences their susceptibility to fire. Let a new variable $h$ denote the product of the woody plant density and the average age of the woody plants. A first approximation yields $d h / d t=w$, but that relationship neglects the influence of fire on the average age. The dynamics of $w$ and $h$ are given by

$$
\begin{aligned}
& \frac{d w}{d t}=r_{w} w\left(1-c_{g w} g-c_{w w} w\right)-f w+a_{w} \\
& \frac{d h}{d t}=w-r_{f} f h
\end{aligned}
$$

where the fire risk $f^{r}$ is defined as follows: let the fire potential $\boldsymbol{\rho}$ be proportional to the available fuel:

$$
\rho= \begin{cases}c_{f g_{f}} & \text { if } h>20 \\ c_{f} g_{f} h / 20 & \text { otherwige. }\end{cases}
$$

We assume that the fire risk $f^{r}$ is given by

$$
f=\rho \frac{h^{\alpha}}{\left(w a_{0}\right)^{\alpha}+h^{\alpha}}
$$

where the parameter $a_{0}$ is an age at which the fire risk is half of its maximum, and the parameter $\alpha$ determines the sharpness of the increase of fire risk with age. We have used $\alpha=9$ to give a sharp increase, and $a_{0}=60$. The remaining parameters were chosen as

$r_{g}=5, c_{w}=.8, c_{g g}=1, a_{g}=.02, c_{f}=.4, r_{w}=3, c_{w w}=.8, c_{w w}=1, a_{w}=.02$.

The stocking rate $s$ may be chosen as a parameter or control variable. In the following, we chose $s=.5$.

Because there are three state variables in this system, one cannot make a meaningful phase plot. However, if the age variable $h$ is fixed, we may gain an impression of the dynamics. Figure 7 shows how a low value of $h$ leads the system to an equilibrium with high $w$. On the other hand, a higher value of $h$ leads to an equilibrium with much lower $w$, as shown in Fig. 8 . Now, if $h$ increases with time, the system will first have high $w$, then lower $w$ as it ages. Fires decrease the average age of trees as well as their density. Hence, the system gets reset to a state analogous to that shown in Fig. 7 after a fire. The cycling of $w$ with time in the full system, with $h$ changing, is shown in Fig. 9 . 
FI G. 7. The phase plane for grass and trees for the system (21)-(28), neglecting the dynamics of the age variable $h$, with a low value of $h$. There is only a single equilibrium with a high value of $w$ and a lower value for 9 , and it is stable. All trajectories approach this equilibrium.

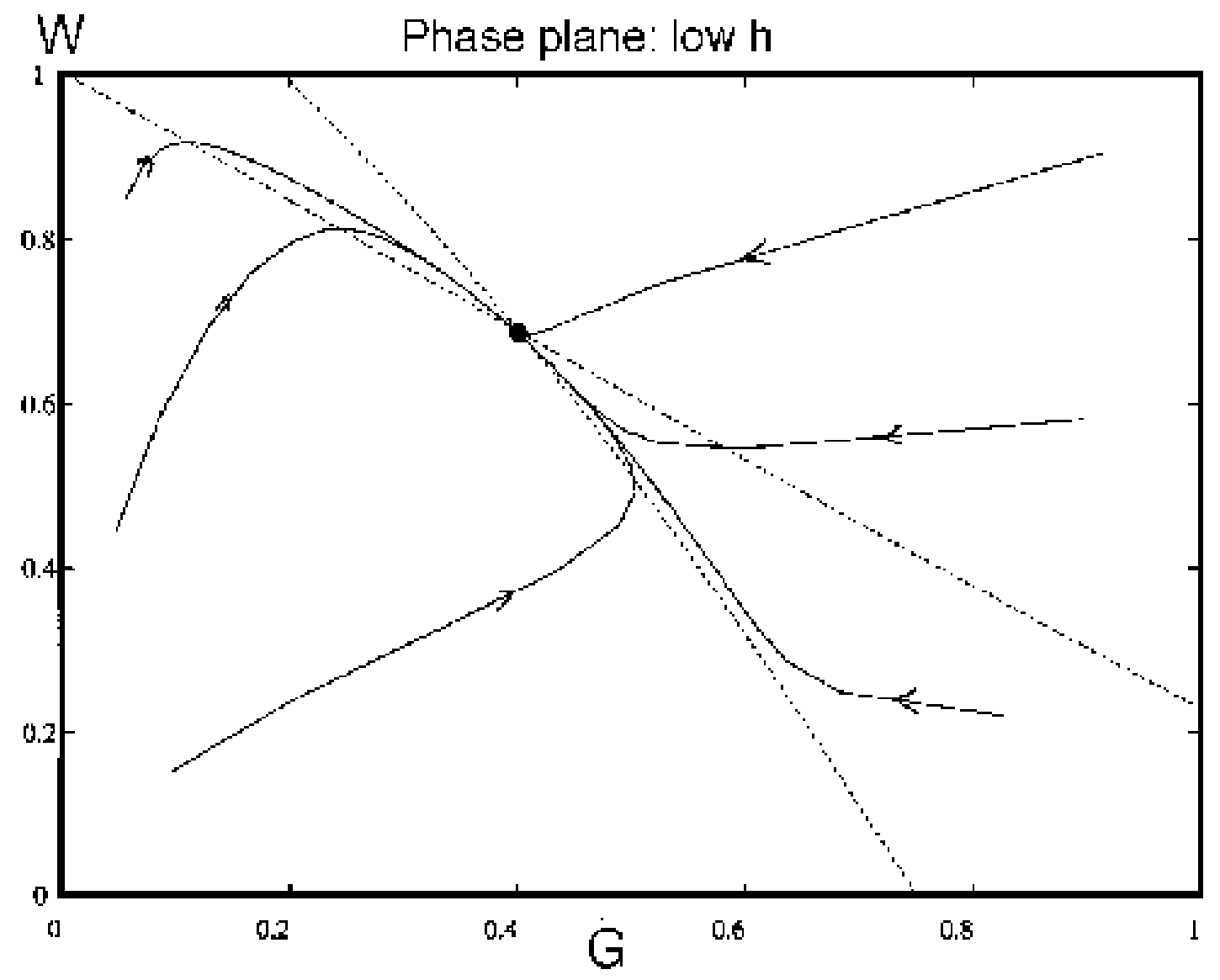

FI G. 8. The phase plane for grass and trees for the system (21)-(28), neglecting the dynamics of the age variable $h$, with a high value of $h$. The single equilibrium has a low value for $w$ and a moderately high value for 9 . 


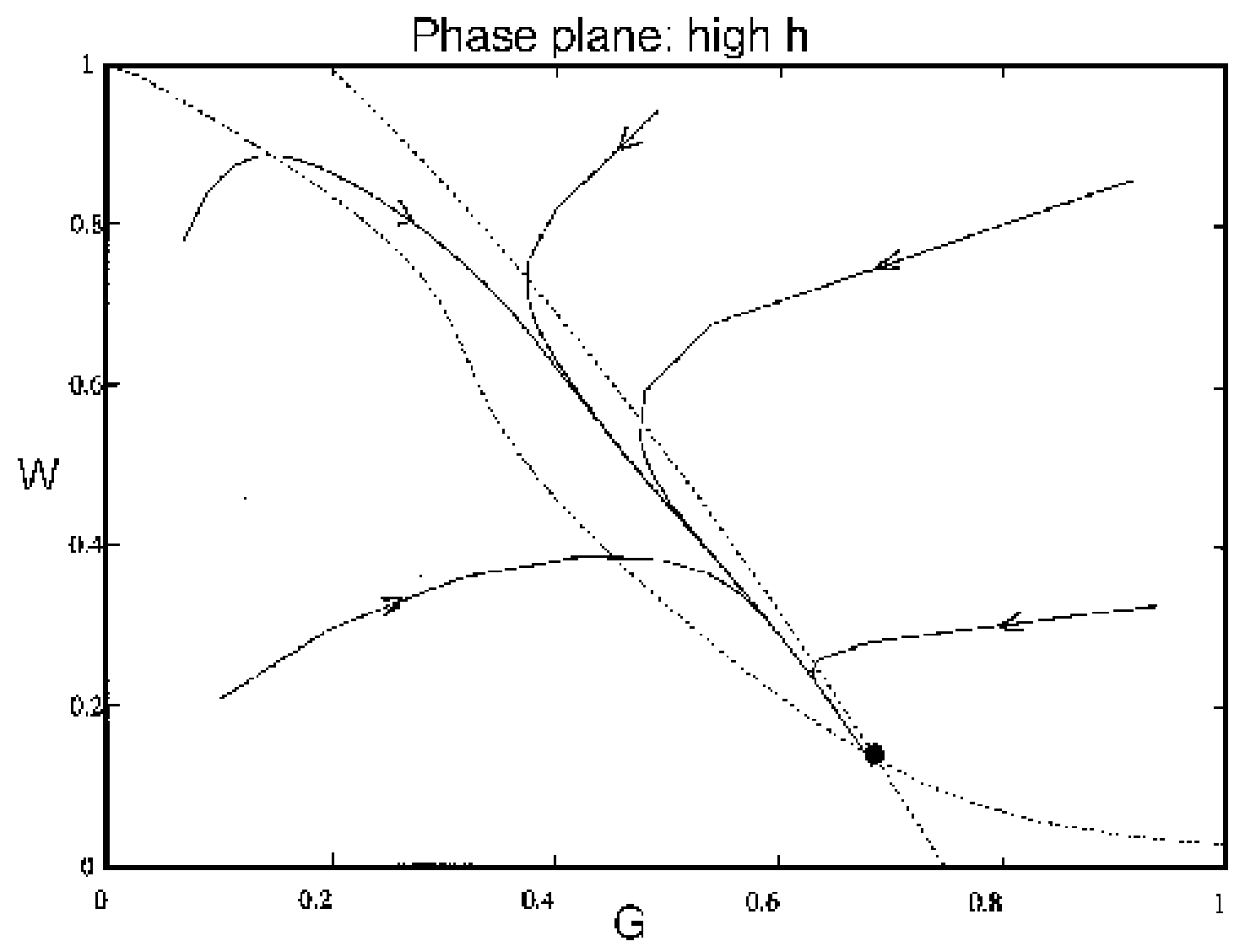

FI G. 9. Tree density (W) vs. time ( $T$, in years) for the system (21)-(28), including dynamics for the age variable. A similar figure could be drawn for the grass density, which tends to be high when woody vegetation is low, and vice versa.

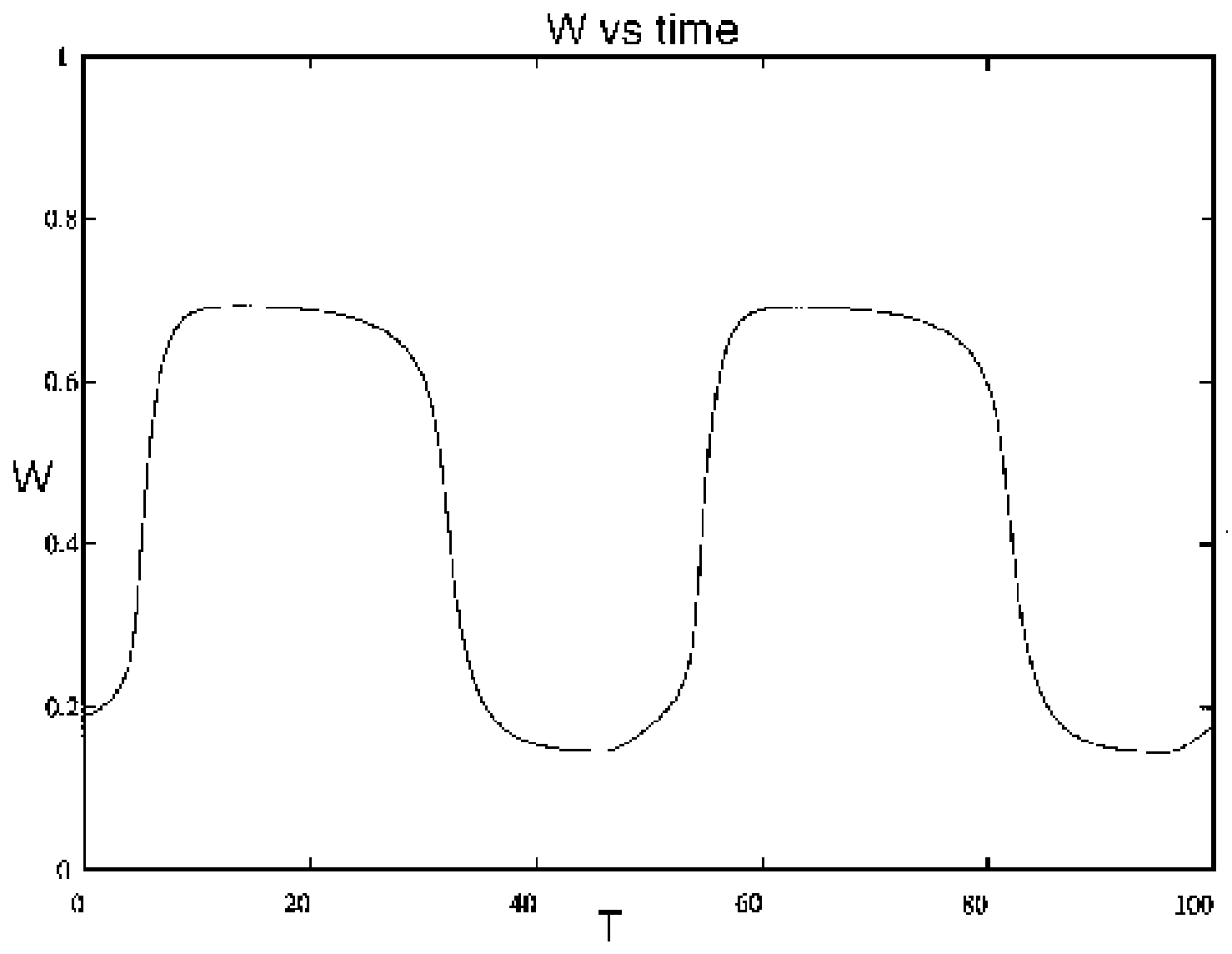


If one were to observe this system over a time of 5-20 years, it would appear that woody plants would eventually dominate grasses because of the combination of competition and grazing. Over the next 30 years, however, the effect of fire and aging of trees leads to a collapse of the trees, making way for the next cycle. This example illustrates how the time scale over which we observe the system may have a decisive influence upon our classification of its behavior. Unfortunately, the scale over which we are able to observe the system is often much shorter than the scale over which it exhibits its characteristic behavior.

\section{CONCLUDI NG REMARKS}

The examples presented here illustrate the complexity of the task facing us as we attempt to clarify the concepts of sustainability and resilience for natural systems. Mathematical theory presents a wealth of possibilities, but the limitations of our understanding and the data available make it difficult to distinguish between them.

Here, we have proceeded from simple conceptual models, such as the raft analogy, to simple but abstract mathematical models, to ecosystem analogues, and finally to a fairly detailed model of a savanna system. In no case can these analogies be considered to be complete, nor is our knowledge of the systems detailed enough to support a full-blown model with statistical justification. Perhaps one might conclude that all this is merely speculation, unworthy of serious attention. On the other hand, prudent decision making requires that we take account of a variety of plausible hypotheses about the responses to our actions. The examples presented here do not encourage complacency about the ability of natural systems to support us and our habits in the lavish fashion we have enjoyed in the past. If we refuse to contemplate possibilities that are only dimly perceived, we may miss the opportunity to learn about the world and adapt our behavior accordingly. If we insist that the simplest and most convenient hypotheses have priority when choosing actions, we run the risk of allowing sloth and pride to bring us to disaster.

\section{RESPONSES TO THIS ARTI CLE}

Responses to this article are invited. If accepted for publication, your response will be hyperlinked to the article. To submit a comment, follow this link. To read comments already accepted, follow this link.

\section{Acknowledgments}

This research was the product of a Resilience Network Planning Workshop supported by the Beijer Institute, a Division of the Royal Swedish Academy of Sciences.

\section{LITERATURE CITED}

Arrow, K., B. Bolin, R. Costanza, P. Dasgupta, C. Folke, C. S. Holling, B.-O. Janssen, S. Levin, K.-. Mäler, C. Perrings, and D. Pimentel. 1995. Economic growth, carrying capacity, and the environment. Science 268: 520521.

Carpenter, S. R., B. O. Jannson, D. Ludwig, J. Pastor, G. Peterson, and B. Walker. 1996. A comparative analysis of total system resilience. Notes from the Beijer Institute Resilience Network Planning Workshop April 1016.

Carpenter, S. R., and J. F. Kitchell. 1993. The trophic cascade in lakes. Cambridge University Press, Cambridge, 
UK.

Carpenter, S. R., and P. R. Leavitt. 1991. Temporal variation in the paleolimnological record arising from a trophic cascade. Ecology $\mathbf{7 2 ( 1 ) : ~ 2 7 7 - 2 8 5 . ~}$

D'Antonio, C. M., and P. M. Vitousek. 1992. Biological invasions by exotic grasses, the grass-fire cycle, and global change. Annual Review of Ecology and Systematics 23: 63-87.

Done, T. J . 1992. Phase shifts in coral reef communities and their ecological significance. Hydrobiologia 247: 121132.

Dublin, H. T., A. R. E. Sinclair, and J. McGlade. 1990. Elephants and fire as causes of multiple stable states in the Serengeti-Mara woodlands. J ournal of Animal Ecology 59: 1147-1164.

Edelstein-Keshet, L. 1988. Mathematical models in biology. Random House, New York, New York, USA.

Estes, J. A., and D. Duggins. 1995. Sea otters and kelp forests in Alaska: generality and variation in a community ecological paradigm. Ecological Monographs 65(1): 75-100.

Guckenheimer, J ., and P. Holmes. 1983. Nonlinear oscillations, dynamical systems, and bifurcations of vector fields. Springer-Verlag, New York, New York, USA.

Harper, D. 1992. Eutrophication of freshwaters. Chapman and Hall, London, UK.

Holling, C. S. 1973. Resilience and stability of ecological systems. Annual Review of Ecology and Systematics 4: 123.

Hughes, T. P. 1994. Catastrophes, phase shifts, and large-scale degradation of a Caribbean coral reef. Science 265: 1547-1551.

J ansson, B.-O., and H. Velner. 1995. The Baltic: the sea of surprises. Pages 292-372 in L. H. Gunderson, C. S. Holling, and S. S. Light, editors. Barriers and bridges to the renewal of ecosystems and institutions. Columbia University Press, New York, New York, USA.

Jones, R. I. 1992. The influence of humic substances on lacustrine planktonic food chains. Hydrobiologia 229: 7391.

Ludwig, D., D. Jones, and C. S. Holling. 1978. Qualitative analysis of insect outbreak systems: the spruce budworm and the forest. Journal of Animal Ecology 47: 315-332.

McClanahan, T. R., A. T. Kamukuru, N. A. Muthiga, M. Gilagabher Yebio, and D. Obura. 1996. Effect of sea urchin reductions on algae, coral, and fish populations. Conservation Biology 10(1): 136-154.

National Research Council. 1992. Restoration of aquatic ecosystems. National Academy Press, Washington, D. C., USA.

Odum, E. P. 1993. Ecology and our endangered life support systems. Second edition. Sinauer, Sunderland, Massachusetts, USA.

Pimm, S. L. 1991. The balance of nature? University of Chicago Press, Chicago, Illinois, USA.

Scheffer, M., S. H. Hosper, M.-L. Meijer, B. Moss, and E. Jeppesen. 1993. Alternative equilibria in shallow lakes. TREE 8(8): 275-279.

Schindler, D. W. 1990. Experimental perturbations of whole lakes as tests of hypotheses concerning ecosystem structure and function. Oikos 57: 25-41. 
Vollenwieder, R. A. 1976. Advances in defining critical loading levels for phosphorus in lake eutrophication. Memorie dell'I stituto Italiano di Idrobiologia 33: 53-83.

Walker, B. H., D. Ludwig, C. S. Holling, and R. M. Peterman. 1981. Stability of semiarid savanna grazing systems. Journal of Ecology 69:473-498.

Zimov, S. A., V. I. Chuprynin, A. P. Oreshko, F. S. Chapin, II I, J . F. Reynolds, and M. C. Chapin.1995. Steppe-tundra transition: a herbivore-driven biome shift at the end of the Pleistocene. American Naturalist 146(5): 765- 794.

\section{APPENDIX}

\section{Return times and resilience}

It is important to distinguish between behavior near a stable equilibrium and behavior near the boundary of a domain of attraction, which is an unstable equilibrium or separatrix. As discussed in Section 2, the long return times associated with a loss of resilience are caused by slow dynamics near the unstable equilibrium, not by slow dynamics near the stable equilibrium point. Unfortunately, there are two conflicting definitions of resilience and consequent confusion about the connection between resilience and return times.

Pimm (1991:13) defines resilience as "how fast a variable that has been displaced from equilibrium returns to it. Resilience could be estimated by a return time, the amount of time taken for the displacement to decay to some specified fraction of its initial value." Pimm (1991: 33) describes return to equilibrium by the equation

$$
X_{t}-X^{*}=\left(X_{0}-X^{*}\right) e^{-k t} \text {, }
$$

where $X_{t}$ is the population density at time $t, X_{0}$ is the initial population density, and $X^{*}$ is the equilibrium density. The differential equation for $\boldsymbol{X}_{t}$ that corresponds to this formula is

$$
\frac{d X_{t}}{d t}=-k\left(X_{t}-X^{*}\right)
$$

A similar model with discrete time could be given instead, but that would not alter the following argument. If we measure displacement from $\boldsymbol{X}^{*}$ by $\boldsymbol{I}$, then $\boldsymbol{I}$ satisfies

$$
\frac{d x}{d t}=-k x
$$

which is equivalent to $\mathrm{Eq}$. (A.1) if $\boldsymbol{h}(\boldsymbol{\alpha})-\boldsymbol{I}$ is replaced by $\boldsymbol{\tau}$. Strictly speaking, Pimm's definition depends upon this simplicity, because the amount of time required for $\mathbb{E}$ to decay to some specified fraction of its initial value is only constant if the model $\left(A_{.1}\right)$ is used. In fact, if the initial displacement is $\mathbb{I}_{0}$ and the fraction is $p<1$, then (A.1) implies that

$$
\boldsymbol{x}_{1}=\mathrm{p} \boldsymbol{x}_{0}=\boldsymbol{x}_{0} e^{-k t_{r}},
$$

from which we conclude that the return time $t_{r}$ is given by

$$
t_{r}=\frac{1}{k} \log \frac{1}{p}
$$

The remarkable feature is that the magnitude $\boldsymbol{I}_{0}$ does not appear in this formula. This is a feature of this model only, as we shall see below. In more general circumstances, such a result can be expected to hold only in the limit as $\mathbb{I}_{0} \rightarrow[$. Such results are called "local." As pointed out in Section 2, a common error is to extrapolate local results to global ones. In the present context, it amounts to replacing a complicated function by a linear approximation. Such approximations are certainly easy to work with, but they may miss essential features of the 
dynamics. In fact, failure to recognize the distinction between local stability and global stability can lead to unwarrented optimism about the likely consequences of interventions in natural systems. If we think that stability to small perturbations necessarily implies stability to large perturbations, then precautions are never required.

In order to distinguish behavior near the equilibrium at $\mathbb{I}=0$ from behavior near an unstable equilibrium, we must use a model with more parameters than $($ A. 2$)$. We set

$$
\frac{d x}{d t}=f_{1}(x)=\frac{x\left(x^{2}-\alpha\right)}{x^{2}\left[\frac{2}{k_{1}}-\frac{1}{k}\right]+\frac{\alpha}{k}} \text {. }
$$

This particular form leads to an especially simple equation for the return time: the time to reach a position $\boldsymbol{I}_{\mathbf{l}}$ starting at $\mathbb{L}_{\mathbb{Q}}$ is given by

$$
t_{r}=\int_{x_{0}}^{x_{1}} d t=\int_{x_{0}}^{x_{1}} \frac{d x}{f_{1}(x)}
$$

and the form for $f_{1}(x)$ was chosen so that

$\frac{1}{f_{1}(x)}=\frac{-1}{k x}+\frac{1}{k_{1}(x-\sqrt{\alpha})}+\frac{1}{k_{1}(x+\sqrt{\alpha})}$

as can be verified algebraically. In view of $\underline{(A .7)}$ and $\underline{(A .8)}$,

$t_{r}=\frac{1}{k} \log \frac{x_{0}}{x_{1}}+\frac{1}{k_{1}} \log \frac{x_{1}-\sqrt{\alpha}}{x_{0}-\sqrt{\alpha}}+\frac{1}{k_{1}} \log \frac{x_{1}+\sqrt{\alpha}}{x_{0}+\sqrt{\alpha}}$

Now, if we replace $\mathbb{L}_{1}$ by $\mathrm{pI}_{0}, \underline{(A .9)}$ becomes

$t_{r}=\frac{1}{k} \log \frac{1}{p}+\frac{1}{k_{1}} \log \frac{1}{p_{1}}+\frac{1}{k_{1}} \log \frac{1}{p_{2}}$,

where

$$
\begin{aligned}
& p_{1}=\frac{x_{0}-\sqrt{\alpha}}{p x_{0}-\sqrt{\alpha}} \\
& p_{2}=\frac{x_{0}+\sqrt{\alpha}}{p x_{0}+\sqrt{\alpha}}
\end{aligned}
$$

If the last two terms in $(\mathrm{A} .10)$ are omitted, this result is identical to Pimm's assumption (A.1). Our more complicated dynamical assumption $(A .6)$ is the analogue of Pimm's assumption if there are three equilibria. Under what conditions does (A.10) imply large return times? The first term, which corresponds to Pimm's model, implies a long return time if the ratio $\boldsymbol{p}=\boldsymbol{x}_{1} / \boldsymbol{x}_{0}$ is small or if $\boldsymbol{k}$ is small. In Pimm's discussion, $\boldsymbol{p}$ is a parameter that describes a probe or observation of the system. Ordinarily, $\mathbf{p}$ is fixed, and the return time provides an estimate for k.

The second term in $(\mathrm{A} .10)$ implies a long return time if $\mathrm{P}_{\mathbf{l}}$ is small or $\boldsymbol{k}_{\mathbf{l}}$ is small. Our previous discussion was concerned with a possibly variable $\alpha$ and disturbances that might take the system near an unstable equilibrium. That corresponds to $\mathbb{I}_{0 \text { near }} \sqrt{\alpha}$, or $\mathbb{x}_{0 \text { near }}-\sqrt{\alpha}$. In such a case, $t_{\boldsymbol{r}}$ will be large even if the parameter $k$ is large. That is, return times may be long, even for systems that show very rapid return when close to the stable equilibrium. According to this point of view, long return times may be diagnostic for a small $\alpha$ or for disturbances that are large enough to take the system near an unstable equilibrium. They may also correspond to weak repulsion from the unstable equilibrium, i. e., small $k_{1}$. If a disturbance takes the system beyond the unstable equilibrium, there is no return at all.

In summary, according to Pimm (1991) and according to us, long return times may be diagnostic for a loss of resilience, but the meanings of the terms are quite different in the two cases. Pimm is concerned with behavior near a stable equilibrium. In that case, a long return time for a given displacement from the equilibrium indicates a small 
coefficient $k$ or, equivalently, a small derivative of $\log x$. We are concerned with behavior of a system with two or three equilibria, one of which is stable. Resilience describes the tendency of the system to return to its stable equilibrium. A long return time is due to disturbances that bring the system near an unstable equilibrium, or possibly to a weak repulsion from an unstable equilibrium.

\section{Address of Correspondent:}

Donald Ludwig

Department of Mathematics

University of British Columbia

Vancouver, British Columbia, Canada V6T $1 Z 2$

phone: 604-541-9409

fax: 604-822-6074

ludwig@math.ubc.ca

\section{Addresses of Coauthors:}

Brian Walker

CSIRO, Division of Wildlife and Ecology

G.P.O. Box 84

Lynham, ACT 2602, Australia

Phone: 6-242-1742

Fax: 6-241-1742

brian.walker@dwe.csiro.au

Crawford S. Holling

University of Florida

Department of Zoology, P.O. Box 118525

Gainesville, Florida 32611 USA

Phone: 352-392-6914

Fax: 352-392-3704

holling@zoo.ufl.edu

${ }^{*}$ The copyright to this article passed from the Ecological Society of America to the Resilience Alliance on 1 January 2000. 